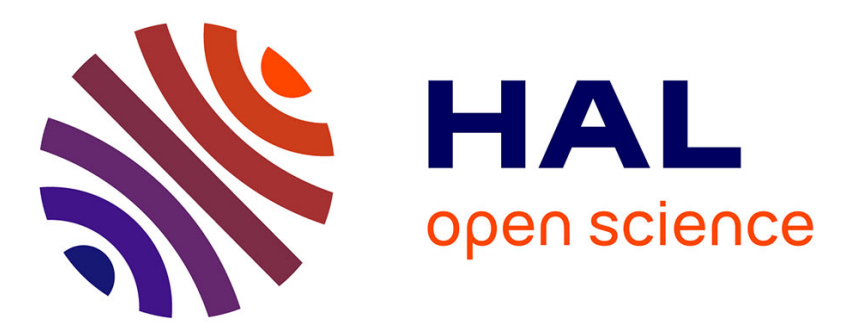

\title{
Modèle d'endommagement micromécanique anisotrope appliqué à des céramiques
}

\author{
C. Aymard (vinet), P. Priou
}

\section{To cite this version:}

C. Aymard (vinet), P. Priou. Modèle d'endommagement micromécanique anisotrope appliqué à des céramiques. Journal de Physique IV Proceedings, 1997, 07 (C3), pp.C3-663-C3-668. 10.1051/jp4:19973113 . jpa-00255398

\section{HAL Id: jpa-00255398 https://hal.science/jpa-00255398}

Submitted on 1 Jan 1997

HAL is a multi-disciplinary open access archive for the deposit and dissemination of scientific research documents, whether they are published or not. The documents may come from teaching and research institutions in France or abroad, or from public or private research centers.
L'archive ouverte pluridisciplinaire HAL, est destinée au dépôt et à la diffusion de documents scientifiques de niveau recherche, publiés ou non, émanant des établissements d'enseignement et de recherche français ou étrangers, des laboratoires publics ou privés. 


\title{
Modèle d'endommagement micromécanique anisotrope appliqué à des céramiques
}

\author{
C. Aymard (Vinet)* and P. Priou
}

\author{
Aérospatiale Espace et Défense, SY/XA, 66 Route de Verneuil, BP.2, 78133 Les Mureaux cedex, France \\ * UFR de Mathématiques, Université de Bordeaux I, 351 Cours de la Libération, 33405 Talence cedex, \\ France
}

Résumé : Cet article propose une modélisation anisotrope de l'endommagement des céramiques soumises à des chargements dynamiques. C'est une généralisation du modèle micromécanique de Rajendran. L'endommagement est représenté par trois systèmes de fissures orthogonales permettant la prise en compte de l'anisotropie induite par le chargement. Des cinétiques de nucléation et de croissance, conduisant à la pulvérisation du matériau ont été adaptées à la nouvelle description statistique des microfissures. Le modèle a été implanté dans le code DYNA3D et appliqué avec succès à la simulation d'essais de compression dynamique aux barres d'Hopkinson.

Abstract : This paper deals with an anisotropic modelisation of ceramic damage under dynamic loading. It's a generalisation of Rajendran's micromechanical model. The damage is represented by three orthogonal cracks systems, allowing the loading - induced anisotropy to be taken into account. A nucleation and a growth kinetic law, implying material pulverisation, are adjusted to a new statistical microcrack description. The model is implanted in the code DYNA3D and applied with success to the simulation of compressive Hopkinson's Bar Tests.

\section{1 - INTRODUCTION}

De nombreuses recherches ont été entreprises ces dernières années sur les matériaux céramiques et composites à matrice céramique, afin d'étendre et d'affiner l'état des connaissances sur leur comportement sous sollicitations dynamiques. Construites à partir des liaisons ioniques et covalentes, les céramiques présentent des caractéristiques mécaniques élevées (dureté, résistance en compression) mais une faible ténacité conduisant à une rupture à caractère fragile. Des études phénoménologiques sur le comportement des céramiques soumises à un impact ont montré l'influence des micromécanismes sur la réponse macroscopique du matériau. Des modifications irréversibles de la microstructure à savoir, apparition et croissance de microfissures ou de microvides se traduisent à l'échelle macroscopique par une diminution des propriétés élastiques du matériau. Un état d'endommagement est décrit à partir de la définition de variables internes représentatives de l'état de détérioration du matériau et on peut distinguer deux approches : les modèles phénoménologiques et les modèles micromécaniques. Les premiers s'appuient sur le concept de contrainte effective [1-5] et sont formulés à partir des potentiels thermodynamiques et dissipatifs. Le champ de déformations inélastiques et les effets des microfissures sont pris en compte de manière approximative en exprimant les potentiels en fonction de variables d'état internes. La détermination de ces variables et des lois cinématiques gouvernant l'évolution de l'endommagement nécessite la mise en place d'expériences. Dans l'approche micromécanique [6-15], les variables d'endommagement sont construites à partir de la description de la microstructure (densité de microfissures ou porosité). La résolution d'un problème de frontières à l'échelle micro, puis l'utilisation d'une technique d'homogénéisation, moyennant la solution sur un volume représentatif, permet de déterminer les équations d'évolution et les propriétés matérielles macroscopiques. Cette approche tend à limiter les expériences macroscopiques visant à définir le comportement mais elle exige des méthodes numériques complexes.

Face aux difficultés d'exploitation des essais dynamiques et au manque de données précises sur la chronologie et l'évolution de l'endommagement des céramiques, on s'est orienté vers une description micromécanique des mécanismes d'endommagement.

Le modèle développé est une généralisation du schéma micromécanique de Margolin/Rajendran [16-18] concernant le comportement élastique effectif d'un matériau microfissuré et traitant la croissance des microfissures jusqu'à leur coalescence, laquelle conduit à une pulvérisation du matériau. Margolin [18] considère une fissuration d'orientation aléatoire générant un comportement isotrope ou des fissures parallèles à un plan donné. La généralisation proposée consiste à prendre en compte l'anisotropie induite 
par le chargement, en définissant trois systèmes orthogonaux de microfissures circulaires, auxquels des cinétiques de nucléation et de croissance ont été adaptées.

Le modèle [19] est décrit dans la suite. Il a été installé dans le code DYNA3D [20] et exploité sur des barres d'Hopkinson avec des comparaisons essais-calculs. Il est aussi comparé avec un modèle fragile.

\section{2 - DESCRIPTION DU MODELE D'ENDOMMAGEMENT}

Dans le modèle [19], l'endommagement du matériau, initialement isotrope, est représenté par la présence de trois systèmes de fissures orthogonales permettant la prise en compte de l'anisotropie induite par le chargement. La diminution des propriétés mécaniques est reliée à des paramètres microscopiques (rayon de microfissures, densité de fissures...). Le modèle comporte les caractéristiques suivantes :

- une distribution de fissures associée à chaque direction,

- une loi de croissance des fissures,

- une définition des paramètres d'endommagement,

- une théorie du module effectif reliant l'état microscopique à l'état macroscopique.

\subsection{Distribution statistique de microfissures}

L'état microscopique du matériau est décrit en introduisant à chargement donné, une relation entre la taille et l'orientation des fissures. On définit pour cela, trois systèmes (populations) de microfissures circulaires et d'épaisseur négligeable, orientées suivant les axes du repère global. Les systèmes sont supposés non évolutifs quant à leur orientation après la nucléation des fissures, ce qui impose que les symétries du matériau endommagé soient prédéfinies. Chaque système de microfissures est caractérisé par une distribution statistique exponentielle des rayons de fissures. Pour chaque population, le nombre de microfissures de normale $\overrightarrow{\mathrm{n}}_{\mathrm{i}}$ et de rayon supérieur à $\mathrm{a}_{\mathrm{i}}$, par unité de volume, est donné par :

$$
\mathrm{N}_{0, \mathrm{i}} \mathrm{e}^{-\frac{\mathrm{a}_{\mathrm{i}}}{\overline{\mathrm{a}}_{\mathrm{i}}}}
$$

L'état d'endommagement est caractérisé par trois paramètres pour chaque système de microfissures, sojert $: \overline{\mathrm{a}}_{i}$ le rayon moyen des fissures de normale $\overrightarrow{\mathrm{n}}_{i}$, a $a_{\max , i}$ le rayon maximal des fissures de normale $\vec{n}_{i}, N_{f, l}$ le nombre de fissures de normale $\vec{n}_{i}$ par unité de volume. Les populations de microfissures sont supposeej évoluer indépendamment les unes des autres et l'interaction entre les fissures est négligée, tant que la densité globale de microfissures $\gamma$ ne dépasse pas une valeur critique.

\subsection{Loi de croissance des microfissures et loi de pulvérisation}

Le mécanisme de croissance des microfissures est établi à partir de la théorie des mécanismes de la ruptuř linéaire dynamique. On distingue la nucléation, la croissance et la coalescence.

- Nucléation des microfissures : Elle s'appuie sur le critère de Griffith généralisé [22-24]. Elle a lieu, pour un volume représentatif donné et pour la microfissure la plus grande associée à ce volume, quand l'état des contraintes satisfait :

$$
\frac{\partial \mathrm{W}}{\partial \mathrm{a}}>\frac{\partial \mathrm{S}}{\partial \mathrm{a}}
$$

avec S, l'énergie surfacique libérée, $\mathrm{W}$, l'énergie de déformation et a, le rayon de la fissure.

Le critère est établi à partir de l'expression des taux de restitution d'énergie, pour chaque population de fissures. On distingue le comportement en traction du comportement en compression en prenant en compte la perte d'énergie due aux frottements des deux bords d'une fissure fermée.

- Cinétique de croissance : Elle est définie à partir d'une loi qui relie l'accroissement du rayon [25] (pour un système) au taux critique de restitution d'énergie, traduisant la propagation d'une fissure sous des 
conditions de chargement dynamique. La vitesse de propagation des microfissures est limitée à la vitesse des ondes de Rayleigh. Elle est de la forme :

$$
\dot{a}_{i}=n_{1}^{ \pm} C_{r}\left(1-\left(\frac{G_{c}}{G_{i}}\right)^{n_{2}}\right)
$$

avec $\dot{a}_{i}$, la vitesse de propagation de la fissure de rayon $a_{i}$ et de normale $\vec{n}_{i}, G_{i}$, le taux de restitution d'énergie de la fissure de rayon maximal de normale $\vec{n}_{i}, C_{r}$, la vitesse des ondes de Rayleigh, $G_{c}$ le taux de restitution d'énergie critique égale à $\mathrm{K}_{\mathrm{lc}}\left(1-v^{2}\right) / \mathrm{E}, \mathrm{E}$ et $v$ étant respectivement le module d'Young et le coefficient de Poisson du matériau non endommagé, $K_{1 c}$ la ténacité et $n_{1}^{-}, n_{1}^{+}, n_{2}$ des constantes à déterminer ( $\mathrm{n}_{1}^{-}$en traction et $\mathrm{n}_{1}^{+}$en compression).

Cette solution reste valide tant que les vitesses matérielles sont inférieures à la vitesse du son dans le matériau.

- Coalescence : Les microfissures en se propageant s'interceptent et conduisent à une pulvérisation du matériau. Le critère de pulvérisation est établi en définissant une densité de fissures critique correpondant à la valeur pour laquelle le rayon moyen atteint la moitié de la distance moyenne interfissure. Le matériau pulvérisé obéit alors à la loi de Mohr - Coulomb.

\subsection{Définition du paramètre d'endommagement.}

Le paramètre d'endommagement dans chaque direction est défini comme étant $\gamma_{i}$, la densité de microfissures de normale $\overrightarrow{\mathrm{n}}_{\mathrm{i}}$.

$$
\gamma_{i}=\int_{0}^{a_{\max , i}}\left(\frac{N_{0, i}}{\bar{a}_{i}}\right) a_{i}^{3} e^{-\frac{a_{i}}{\bar{a}_{i}}} d a_{i}
$$

Il est proportionnel à $\mathrm{Na}^{3}[18]$. Le terme $\overline{\mathrm{a}}_{j}$ permet de normaliser la densité de probabilité.

\subsection{Théorie du module effectif ou endommagé}

Elle établit le lien entre les descriptions microscopiques et les pertes de rigidité du matériau [18]. A l'aide d'une technique d'homogénéisation permettant de relier des variables microscopiques aux champs macroscopiques et en prenant en compte une déformation additionnelle relative à la présence des microfissures, on obtient l'expression du tenseur des déformations macroscopiques $\mathrm{E}_{\mathrm{ij}}$ en fonction du tenseur des déformations microscopiques $\varepsilon_{\mathrm{ij}}$ :

$$
E_{i j}=\frac{1}{V}\left[\int_{V} \varepsilon_{i j} d v+\frac{1}{2} \int_{S_{c}}\left(n_{i} \cdot u_{j}+n_{j} \cdot u_{i}\right) d S\right]
$$

avec $V$ le volume considéré, $S_{c}$ la surface des microfissures, $u_{i}$ les coordonnées du champ de déplacement, $\mathrm{n}_{\mathrm{i}}$ les coordonnées des normales sortantes aux fissures.

Le tenseur des contraintes moyennes $\bar{\sigma}_{\mathrm{kl}}$ étant équivalent à celui des contraintes macroscopiques $\Sigma_{\mathbf{k} \mathbf{l}}$ appliquées au matériau [21], on a alors

$$
E_{i j}=S_{i j k l}^{0} \bar{\sigma}_{k l}+\alpha_{i j}
$$

avec $\alpha_{\mathrm{ij}}$ le tenseur désignant la déformation additionnelle due aux fissures et $S_{\mathrm{ijkl}}^{0}$, la matrice de souplesse non endommagée.

L'expression des champs solutions est obtenue en formulant les sauts de déplacements d'une fissure à partir de la théorie des fissures en milieu infini. L'influence des composantes du champ de contraintes à l'infini est traduite en termes de champs de déplacements en bord de fissures. La théorie de Margolin, 
exprimant les modules effectifs, est étendue à une distribution statistique des rayons de microfissures dans trois directions orthogonales.

\subsection{Nouveauté du modèle}

La nouveauté du modèle réside dans la modélisation 3D de l'endommagement. Une population de défauts est définie à partir d'une distribution statistique des rayons des microfissures suivant trois directions orthogonales. Les microfissures sont supposées prééxistantes mais non actives jusqu'à la vérification du critère de nucléation. La plupart des modèles micromécaniques [9-11, 16-17] conservent le caractère isotrope du matériau endommagé, indépendamment du chargement en définissant une distribution des fissures de manière aléatoire dans un élément de volume. Le présent modèle permet de décrire un endommagement anisotrope en reliant la croissance des microfissures à la direction du chargement.

\section{APPLICATION}

\subsection{Description du matériau}

Le modèle a été appliqué à une céramique renforcée par des trichytes de $\mathrm{SiC}$. Des micrographies, réalisees en Aquitaine ont montré l'importance d'amas de trichytes et de blocs compacts de SiC, entrainant de nombreux vides dans la structure pour des matériaux à fort pourcentage d'inclusions. La porosité est la défaut le plus critique vis à vis des performances à rupture du composite et les amas de trichytes sont des défauts initiateurs de la rupture. La forte cohésion fibres/matrice au sein du matériau nous a conduit a considérer un matériau équivalent homogène, présentant les mêmes caractéristiques mécaniques que le $\mathrm{SiC} / \mathrm{SiAlYON}$ avec une distribution statistique de microvides traduisant la présence des amas. Le modele d'endommagement développé a donc été utilisé pour simuler le comportement de la céramique soumis a des sollicitations dynamiques.

\subsection{Simulations des essais aux barres d'Hopkinson}

Des essais de compression dynamique aux barres d'Hopkinson ont été réalisés au CREA, en vue d'un: caractérisation dynamique du comportement du SiC/SiAlYON. Ils ont été simulés avec le code DYNA3D à l'aide du modèle d'endommagement et d'un modèle fragile macroscopique construit à partir des contraintes à rupture données par les essais. Les courbes contraintes-déformations ont été comparées aus. essais.

L'utilisation du modèle d'endommagement nécessite de déterminer un certain nombre de paramètres. Les constantes matérielles $\left(\rho, E, v, K_{\mathrm{Ic}}, \mu\right)$ sont issues des essais statiques de caractérisation réalisés en Aquitaine. Les rayons moyen et maximal des microfissures sont estimés à partir de la taille des grains et des amas. Le nombre de fissures par unité de volume correspond à la porosité du matériau comprise entre 2 et $3 \%$. Les paramètres de croissance $\mathrm{n}_{1}^{-}, \mathrm{n}_{1}^{+}, \mathrm{n}_{2}$, qui sont des constantes matérielles à déterminer, ont été approchés après une étude de l'influence de ces paramètres sur l'endommagement du matériau et la simulation d'un des essais aux barres d'Hopkinson. Les tableaux 1 et 2 donnent les valeurs utilisées.

\begin{tabular}{|c|c|c|c|c|c|c|c|c|c|c|c|}
\hline$\rho\left(\mathrm{kg} / \mathrm{m}^{3}\right)$ & $\mathrm{E}(\mathrm{GPa})$ & $v$ & $\mathrm{~K}_{1 \mathrm{c}}(\mathrm{MPa} \sqrt{\mathrm{m}})$ & $\mu$ & $\mathrm{N}_{0}$ & $\mathrm{n}_{2}$ & $\mathrm{n}_{1}^{+}$ & $\mathrm{n}_{1}^{-}$ & $\mathrm{C}_{\mathrm{r}}(\mathrm{cm} / \mu \mathrm{s})$ & $\mathrm{a}_{\max }(\mathrm{cm})$ & $\mathrm{a}_{\operatorname{moy}}(\mathrm{cm})$ \\
\hline 3200 & 370 & 0,25 & 8,3 & 0,24 & $2 \%$ & 0,5 & 1,0 & 0,5 & 0,429 & 0,002 & 0,0003 \\
\hline
\end{tabular}

Tableau 1 : Paramètres du modèle d'endommagement

\begin{tabular}{|c|c|}
\hline$\sigma_{\text {rc }}$ (compression)(MPa) & $\sigma_{\text {rt }}$ (traction)(MPa) \\
\hline 3480 & 320 \\
\hline
\end{tabular}

Tableau 2 : Contraintes à rupture de la loi fragile 
Dans l'essai présenté, le projectile impacte la barre d'entrée à une vitesse de $18,1 \mathrm{~m} / \mathrm{s}$. Les comparaisons essai/calculs, présentés figure 1 montrent l'intérêt du modèle d'endommagement face à la loi fragile macroscopique, basée pourtant sur des valeurs expérimentales. Les critères macroscopiques en contraintes ne semblent pas décrire correctement la rupture de l'éprouvette. Par contre le modèle d'endommagement est capable de reproduire les essais : le matériau céramique récupéré n'est plus qu'une poudre, conformément à l'essai. Une analyse événementielle du comportement de la céramique avec la loi d'endommagement montre que cette pulvérisation des éléments n'est pas uniquement due au passage de l'onde incidente dans l'échantillon mais aussi au retour des ondes transverses. Cette conclusion est obtenue grâce à la modélisation 3D de la fissuration et elle permet d'en déduire que la rupture de 1 'éprouvette n'a pas lieu en compression uniaxiale pure. Une modélisation ID de l'essai pour les céramiques ne serait donc pas suffisante et l'utilisation d'un modèle endommagement anisotrope unilatéral semble iustifiée.
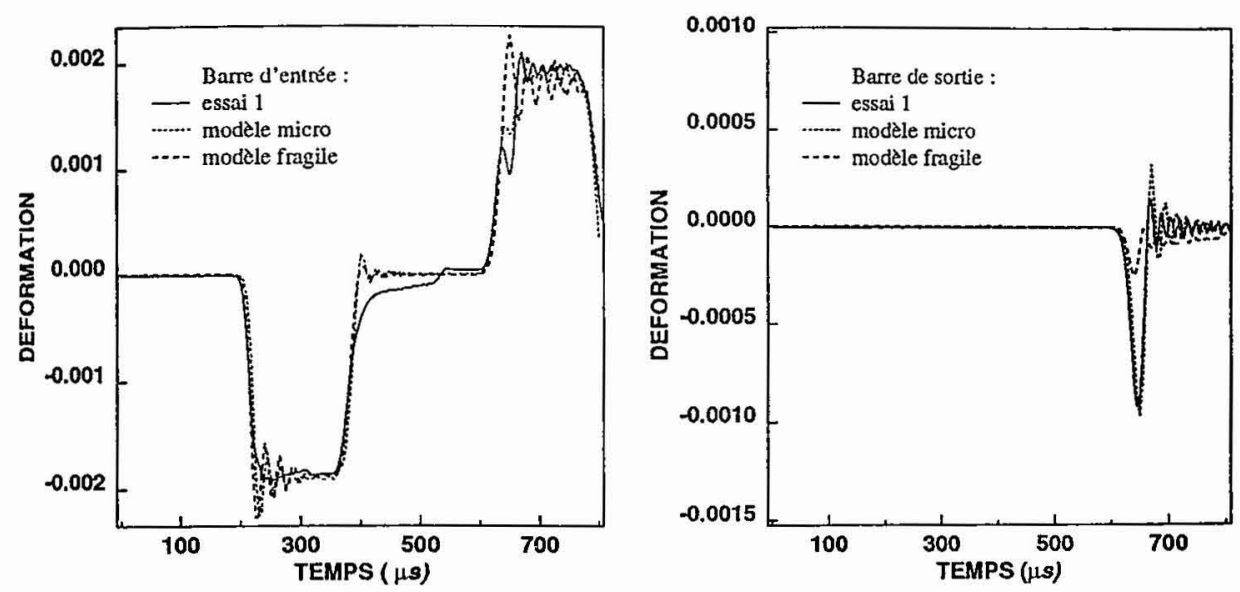

Figure 1 : Comparaison des résultats expérimentaux pour le premier essai. Réponse macroscopique de l'éprouvette lue sur les barres: Déformation en fonction du temps.

Le modèle d'endommagement a ensuite été testé sur un autre essai au barres d'Hopkinson sans rupture de l'échantillon, pour une vitesse du projectile de $13,5 \mathrm{~m} / \mathrm{s}$, terminant ainsi la validation du modèle. Dans ce cas, une bague bloque latéralement l'échantillon cylindrique limitant la déformation à $2 \%$. L'échantillon récupéré est légérement fragmenté suivant la génératrice du cylindre après plusieurs passages de l'onde. La simulation confirme la non-pulvérisation de l'éprouvette et aucun endommagement n'est décelé lors du premier passage. La réponse macroscopique (figure 2) dans les barres est très proche de celle des essais.

Le dépouillement des barres d'Hopkinson sur les céramiques ne permet pas de quantifier l'endommagement de l'éprouvette. Les réponses macroscopiques de l'échantillon, à travers les barres d'entrée et de sortie, sont les seuls résultats disponibles par l'essai. Le modèle microscopique développé offre une possibilité d'exploitation du comportement $3 \mathrm{D}$ réel du matériau.

\section{CONCLUSION}

La généralisation du modèle micromécanique de Rajendran à une description $3 \mathrm{D}$ de l'endommagement des matériaux céramiques permet, dans des axes prédéfinis, de comprendre et de quantifier les différents mécanismes responsables de la rupture du matériau. Les barres d'Hopkinson semblent être une bonne application. Les valeurs des contraintes à rupture macroscopiques, issues des essais, ne sont pas suffisantes pour caractériser le comportement du matériau fragile. L'approche micromécanique, définissant des lois de croissance et de saturation de trois systèmes de fissures offre en contre partie des résultats satisfaisants. 

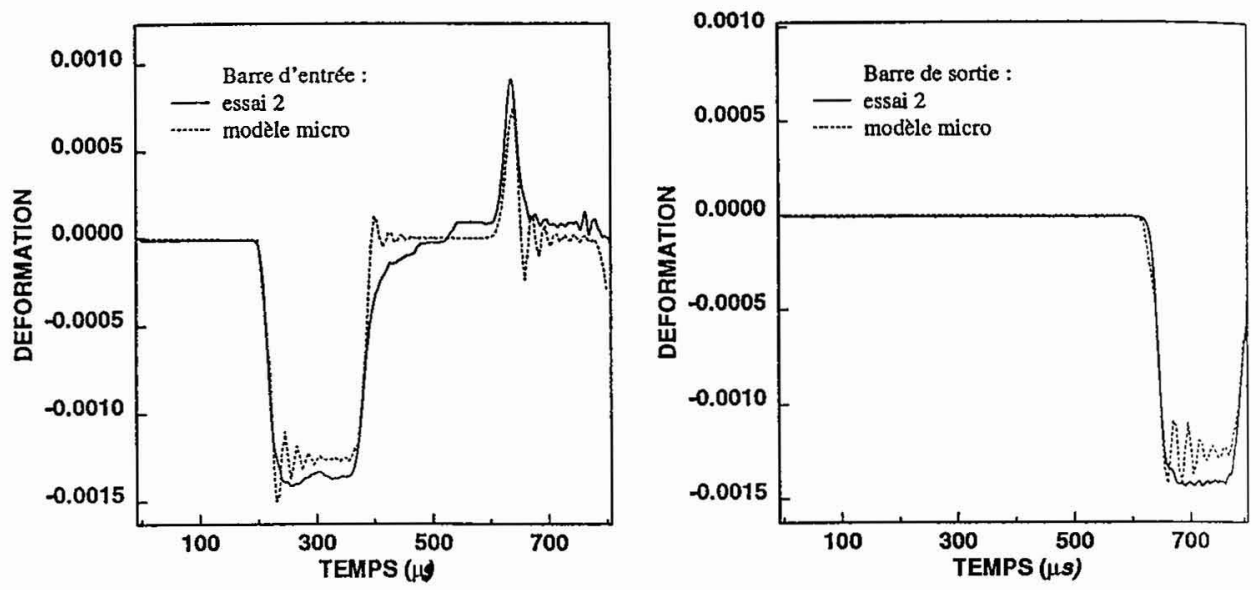

Figure 2 : Comparaison des résultats expérimentaux pour le second essai. Réponse macroscopique de l'éprouvette lue sur les barres : Déformation en fonction du temps.

\section{REFERENCES :}

[1] Kachanov L.M., Izv. Akad. Nauk SSSR, Otd. Tekhn. Nauk No 8, (1958), p. 26

[2] Chaboche J.L.,C. R. Acad. Sci. Paris t. 314 série II, (1992), p. 1395-1401

[3] Chaboche J.L., La Recherche Aérospatiale, No 2 (1995),p. 139-147

[4] Le Maitre J., A course on damage Mechanics. Springer, Verlag, Berlin, 92

[5] Le Maitre J., Chaboche J.L., Mécanique des Matériaux solides, Dunod 85

[6] Seaman L., Curran D.R., Shockey D. A, J. of Applied Physics, Vol. 47, No. 11, 1976

[7] Marigo J.J., C. R. Acad. Sci. Paris, 292, série III, p 1309-1312, 1981

[8] Andrieux S., Bamberger Y., Marigo J.J., J. Méca. Théo. et App., Vol. 5, No 3, (1986) p 471

[9] Taylor L.M., Chen E.P., Kuszmaul J.S., App. Mech. and Engng.55 (1986) p.301-320

[10] Budiansky B., O'Connell R.J., Int. J. Solids Structures, Vol. 12 (1976) p. 81-97

[11] Horii H., Nemat-Nasser S., J. Mech. Phys. Solids, Vol. 31, No. 2, (1983) p. 155-171

[12] Rajendran A. M., Kroupa J.L., J. Appl. Phys. 66, (1989) p. 3560-3565

[13] Baptiste D., Ecole CREA-EDF-INRIA, Modélisation et analyse qualitative de l'endommagement des matériaux et des structures, Clamart, (1990), p.97-117

[14] Tranchet J.Y., Comportement de deux matériaux fragiles polycristallins sous l'effet de la propagation d'une onde sphérique divergente, (1994), Thèse à Bordeaux I.

[15] Addessio F.L., Johnson J.N., J. Appl. Phys. 67 (7), 1 ,(1990), p.3275

[16] Rajendran A. M., AD-A252 979 Wright Laboratory WL-TR-92-4006 (1992) ,p. 4.1

[17] Rajendran A. M., ARL-TR-137,(1993)

[18] Margolin L.G., Int. J. Fract. 22, pp. 65-79,1983

[19] Vinet C., Priou P., AST, No 1, (1997) p. 65-75

[20] Hallquist J.O., Whirley R.G., Dyna3d Users'manual, UCID 19592, Rev5 (1989)

[21] Bui H.B., Van K.D., Stolz C., C. R. Acad. Sc. Paris, t. 292, serie II, (1981), p. 863

[22] Margolin L.G., Int. J. Engng Sci. 22, pp.1171-1179, 1984

[23] Dienes J.K., J. Geophys. Res.88, No. B2 (1983), p. 1173-1179

[24] Dienes J.K, Eng. Fracture Mechanics Vol. 3 No 23 (1986) p. 615-617

[25] Kaninen M.F., Popelar C.H., Advanced Fracture Mechanics, (1985), Oxford University Press 